\title{
Acylglycerol kinase promotes cell proliferation and tumorigenicity in breast cancer via suppression of the FOXO1 transcription factor
}

\author{
Xi Wang ${ }^{1,2+}$, Chuyong Lin ${ }^{1+}$, Xiaohui Zhao ${ }^{1 \dagger}$, Aibin Liü ${ }^{3}$, Jinrong Zhu', Xinghua $\mathrm{Li}^{1}$ and Libing Song ${ }^{1 *}$
}

\begin{abstract}
Background: Acylglycerol kinase (AGK) is reported to be overexpressed in multiple cancers. The clinical significance and biological role of AGK in breast cancer, however, remain to be established.

Methods: AGK expression in breast cancer cell lines, paired patient tissues were determined using immunoblotting and Real-time PCR. 203 human breast cancer tissue samples were analyzed by immunochemistry (IHC) to investigate the relationship between AGK expression and the clinicopathological features of breast cancer. Functional assays, such as colony formation, anchorage-independent growth and BrdU assay, and a xenograft tumor model were used to determine the oncogenic role of AGK in human breast cancer progression. The effect of AGK on FOXO1 transactivity was further investigated using the luciferase reporter assays, and by detection of the FOXO1 downstream genes.

Results: Herein, we report that AGK was markedly overexpressed in breast cancer cells and clinical tissues. Immunohistochemical analysis showed that the expression of AGK significantly correlated with patients' clinicopathologic characteristics, including clinical stage and tumor-nodule-metastasis (TNM) classification. Breast cancer patients with higher levels of AGK expression had shorter overall survival compared to patients with lower AGK levels. We gained valuable insights into the mechanism of AGK expression in breast cancer cells by demonstrating that overexpressing AGK significantly enhanced, whereas silencing endogenous AGK inhibited, the proliferation and tumorigenicity of breast cancer cells both in vitro and in vivo. Furthermore, overexpression of AGK enhanced G1-S phase transition in breast cancer cells, which was associated with activation of AKT, suppression of FOXO1 transactivity, downregulation of cyclin-dependent kinase inhibitors $p 21^{\text {Cip } 1}$ and $p 27^{\text {Kipl }}$ and upregulation of the cell cycle regulator cyclin D1.
\end{abstract}

Conclusions: Taken together, these findings provide new evidence that AGK plays an important role in promoting proliferation and tumorigenesis in human breast cancer and may serve as a novel prognostic biomarker and therapeutic target in this disease.

Keywords: AGK, Breast cancer, Tumorigenicity, FOXO1

\section{Background}

Breast cancer is the most common malignancy and leading cause of cancer-related deaths in females worldwide [1]. Several lines of evidence showed that multiple proteins are dysregulated in primary tumors and are associated with the development and progression of breast cancers [2-4].

\footnotetext{
* Correspondence: Ib.song1@gmail.com

${ }^{\dagger}$ Equal contributors

'State Key Laboratory of Oncology in Southern China, Department of Experimental Research, Sun Yat-sen University Cancer Center, Guangzhou, Guangdong 510060, China

Full list of author information is available at the end of the article
}

Therefore, understanding the roles and molecular mechanisms of these proteins may provide new insights into the physiology and pathology of cancer and enable the development of novel and effective anticancer therapeutics.

FOXO1 is a member of the forkhead box-containing O subfamily (FOXO) family of transcription factors, which play vital roles in a variety of biological processes, including cell cycle arrest, cell death, apoptosis, stress response, cellular differentiation and metabolism $[5,6]$. For instance, ectopically expressing FOXO1 transcriptionally upregulates cell-cycle inhibitors, such as $p 21^{C i p 1}$ and 
$p 27^{\text {Kip } 1}$, and downregulate cell-cycle regulators, such as cyclin D1 and cyclin D2, which result in G1/S arrest of cells [7-9]. Activation of FOXO1 could induce apoptosis through inducing expression of pro-apoptotic proteins, such as Puma, Bim, TRAIL and Fas ligand (FasL) [10-13]. FOXO1 has also been implicated in DNA repair mechanisms through upregulation of GADD 45 a by directly binding to the GADD45 promoter [14]. Conversely, FOXO1 expression is found to be downregulated in multiple human cancers, including prostate cancer, endometrial carcinoma, glioblastoma and breast cancer [15-18]. Therefore, FOXO1 is considered to be as a putative tumor suppressor, and better understanding of the mechanisms that regulate FOXO1 activity may provide clues of novel targets for therapeutic intervention.

Acylglycerol kinase (AGK) is found to be abundantly expressed in the heart, muscle, kidney and brain [19]. By acting as a lipid kinase, it catalyzes the phosphorylation of acylglycerols to generate lysophosphatidic acid (LPA) [19-22], which is a potent lipid mediator that regulates a number of biological processes [23-25]. Recently, AGK is reported to be overexpressed in prostate cancer and esophageal squamous cell carcinoma (ESCC) [19,26,27]. Bektas et al. reported that AGK was upregulated in prostate, uterine, cervical and stomach cancers, and induced proliferation and migration in prostate cancer cells [19]. Chen et al. showed that overexpression of AGK promoted stem cell-like phenotypes in human ESCC both in vivo and in vitro and was correlated with progression and poor prognosis in ESCC [26]. In addition, Nouh et al. found that AGK expression was significantly correlated with primary Gleason grade of prostate cancer foci and prostate capsular invasion [27]. These findings have provided substantial evidence to show that AGK might contribute to the progression and development of cancer. However, the clinical significance and biological role of AGK in human breast cancer remain unclearly.

In this study, we found that AGK was markedly overexpressed in breast cancer cells and clinical tissue samples. Overexpressing AGK dramatically promoted the proliferation and tumorigenicity of breast cancer cell both in vitro and in vivo, whereas silencing AGK had the converse effect. Taken together, our findings suggest that AGK functions as an oncoprotein during breast cancer progression.

\section{Results}

AGK overexpression correlates with progression and poor prognosis in breast cancer

Western blotting and real-time PCR analyses were performed and showed that AGK mRNA and protein expression were markedly upregulated in all tested breast cancer cell lines compared to primary normal breast epithelial cells (NBECs) (Figure 1A and Additional file 1: Figure S1A). Consistently, we found that AGK expression was higher in eight human breast cancer tissues than the paired adjacent non-tumor tissues (Figure $1 \mathrm{~B}$ and Additional file 1: Figure S1B). These results indicate that AGK expression is upregulated in breast cancer.

To investigate the relationship between AGK expression and the clinicopathological features of breast cancer, 203 human breast cancer tissue samples were analyzed by IHC. Consistently, IHC analysis indicated that AGK was markedly upregulated in breast cancer samples (Figure 1C). Furthermore, statistical analysis of the results revealed that AGK expression was strongly associated with the clinical stage $(P<0.001)$, T classification $(P<0.001)$, N classification $(P<0.001)$ and $M$ classification $(P=0.003)$ (Additional file 2: Table S2). Kaplan-Meier survival curves and logrank test showed that AGK expression was significantly correlated with overall survival (OS) in breast cancer $(P<0.001$; Figure 1D). Similar results were obtained between patients in clinical stage I-II and III-IV subgroups (Additional file 3: Figure S2A-B). Furthermore, univariate and multivariate analyses indicated that clinical TNM classification and AGK expression were independent prognostic factors in breast cancer (Additional file 2: Table S3), suggesting that AGK might serve as a prognostic indicator of survival in patients with breast cancer.

\section{AGK promotes the proliferation of breast cancer cells}

Since AGK expression was correlated with clinical stage in breast cancer $(P<0.001$; Additional file 2: Tables S2 and S3), we then examined the relationship between AGK and Ki-67 expression. As shown in Figure 2A and Additional file 2: Tables S2, the tumor areas with the high levels of AGK staining also showed strong Ki-67 expression, whereas areas with low AGK staining intensities exhibited weak Ki-67 signals $(P<0.001)$, suggesting that AGK might promote proliferation of breast cancer cells.

To further investigate the effect of AGK on the proliferation of breast cancer cells by examining gain and loss of function models (Figure 2B). MTT and colony formation assays showed that the proliferation rate of AGK-overexpressing cells was significantly higher than the corresponding vector-control cells, whereas silencing AGK drastically reduced cell proliferation (Figure 2B-F). These results provide strong evidence that AGK plays a critical role in the proliferation of breast cancer cells.

\section{AGK promotes the tumorigenicity of breast cancer cells both in vitro and in vivo}

Next, we investigated the effect of AGK on the tumorigenicity of breast cancer cells. As shown in Figure 3A, upregulation of AGK significantly increased, whereas downregulation of AGK decreased, the anchorage-independent growth ability of MCF-7 and SKBR3 cells in soft agar. Similarly, we observed that MCF-7/AGK tumors 


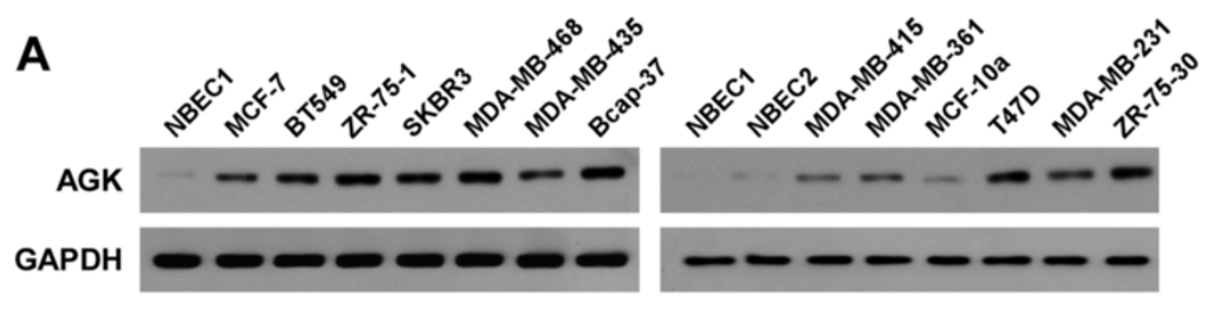

B

Patient
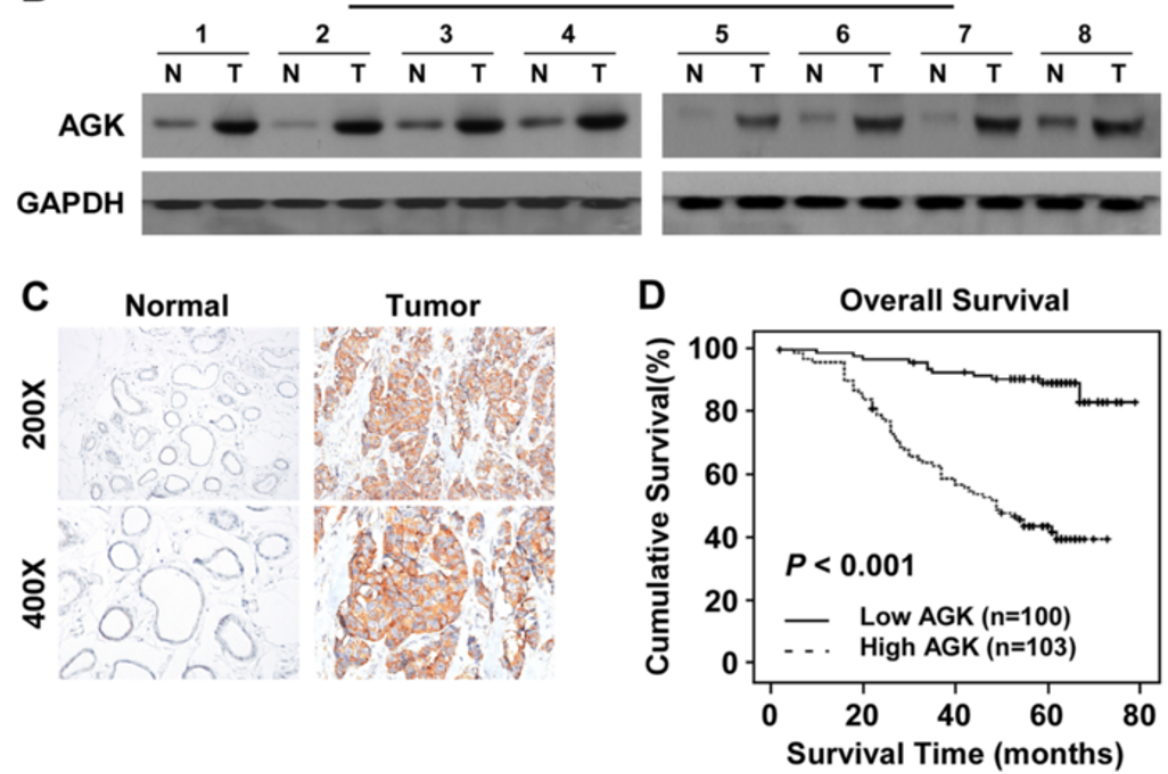

Figure 1 AGK is upregulated in breast cancer. (A and B) Western blotting analysis of AGK expression in normal breast epithelial cells (NBECs) and 12 breast cancer cell lines (A) and in eight matched primary breast cancer tissues (T) and adjacent noncancerous tissues (N) (B). GAPDH was used as a loading control. (C) Immunohistochemical analysis of AGK protein expression in normal breast and primary tumor tissues. (D) Kaplan-Meier overall survival curves and univariate analyses (log-rank) comparing breast cancer patients with low $(n=103)$ and high $(n=100)$ AGK-expressing tumors $(P<0.001)$.

grew significantly faster than MCF-7/vector tumors, whereas the tumors formed by MCF-7/AGK-RNAi cells grew at a much slower rate than control MCF-7/Scrambled-vector tumors (Figure 3B-D). Collectively, these results indicate that AGK plays a significant role in the tumorigenicity of breast cancer cells both in vitro and in vivo.

\section{AGK regulates the G1-S phase transition in breast cancer cells}

To further explore the mechanism by which AGK promotes proliferation of breast cancer cells, BrdUrd incorporation and flow-cytometry assays were performed. As shown in Figure 4A-D, overexpressing AGK significantly increased, but silencing AGK reduced, the percentage of S phase cells. Further analyses by real-time RT-PCR and Western blotting showed that the expression of cyclin dependent kinases (CDK) inhibitors p $21^{\text {Cip } 1}$ and p $27^{\mathrm{Kip} 1}$, at both mRNA and protein levels, were drastically reduced in AGK-overexpressing cells compared to control cells.
This was accompanied by a concurrent increase in the levels of cell cycle regulators cyclin D1 and $\mathrm{p}-\mathrm{Rb}$ (Figure 5A,C). Conversely, the expression of p21 ${ }^{\mathrm{Cip} 1}$ and $\mathrm{p} 27^{\mathrm{Kip} 1}$ significantly increased, whereas expression of cyclin D1 and $\mathrm{p}-\mathrm{Rb}$ decreased, in AGK-silenced cells (Figure 5B-C). Collectively, our results suggest that AGK promotes cell cycle G1/S transition in breast cancer cells.

\section{AGK downregulates FOXO1 transactivity and activates AKT signaling pathway}

Previous reports have demonstrated that FOXO1 transcriptionally regulates $p 21^{\text {Cip } 1}, p 27^{\text {Kip } 1}$ and cyclin D1 $[7,8]$, which prompted us to investigate whether AGK targeted these genes by modulating the transactivity of FOXO1. As shown in Figure 6A-B, the transactivity and expression level of FOXO1 significantly decreased in AGK-overexpressing cells and increased in AGK-silenced cells. We also found that AGK expression inversely correlated with FOXO1 in the ten freshly collected clinical 


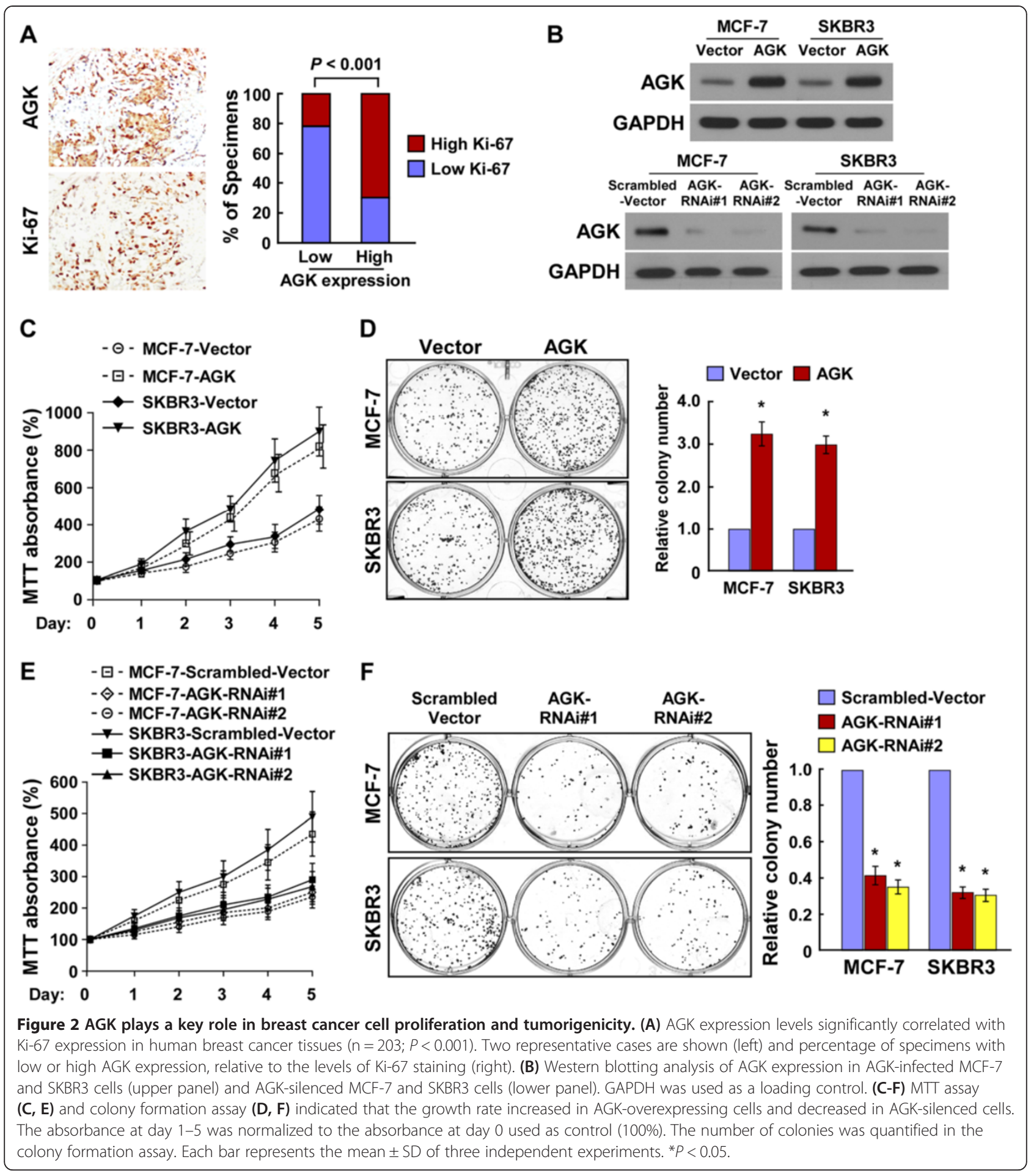

breast cancer samples $(r=-0.721, P=0.019$; Figure $6 C)$. AKT kinase is known to play a key role in phosphorylating and repressing FOXO transcriptional activity [8]. As predicted, overexpressing AGK drastically increased, but silencing AGK decreased, the level of p-AKT and p-GSK$3 \beta$ (Figure $6 \mathrm{~B}$ ), suggesting that AGK contributes to modulation of AKT/FOXO1 and AKT/GSK-3 $\beta$ signalings.
We further examined the role of FOXO1 in AGKmediated cell proliferation. As shown in Additional file 4: Figure S3A, the luciferase activity of the FOXO1 reporter was significantly decreased in AGK-silenced cells after FOXO1 siRNA(s) transfection. Additional knockdown of FOXO1 in AGK-silenced cells decreased p27 ${ }^{\text {Kip1 }}$ and $\mathrm{p} 21^{\mathrm{Cip} 1}$ expression but increased cyclin D1 expression 


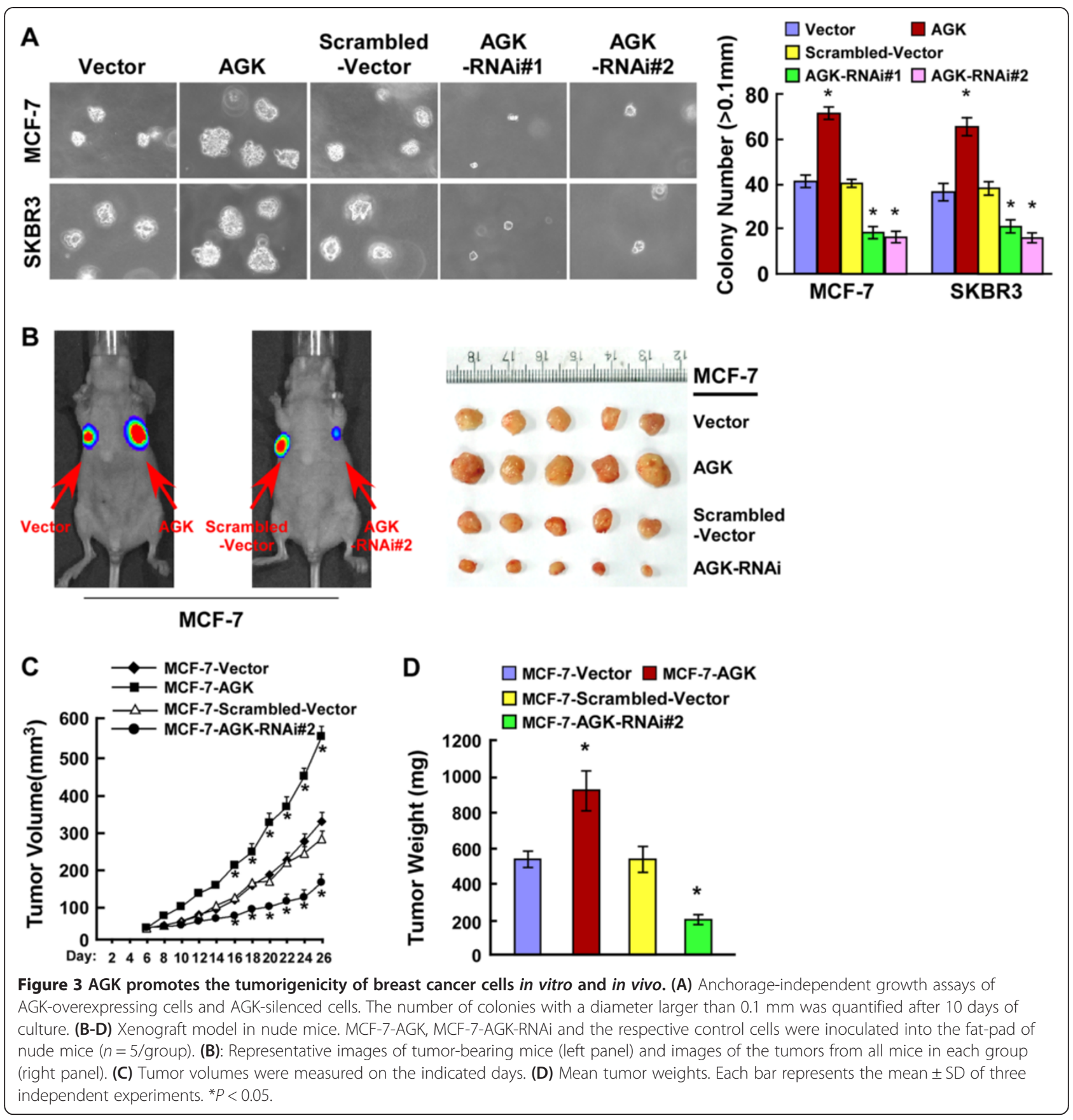

(Figure 6D and Additional file 4: Figure S3B). Furthermore, MTT and colony formation assays showed that silencing FOXO1 restored the growth rate of AGK-silenced cells (Figure 6E-F and Additional file 4: Figure S3C). Taken together, our results suggest that FOXO1 plays a critical role in the pro-proliferative effect of AGK on breast cancer cells.

\section{Discussion}

The key findings presented in this study suggest that AGK is markedly upregulated in breast cancer cells and its ectopic expression promotes the proliferation and tumorigenicity of breast cancer cells both in vitro and in vivo. The mechanistic basis for the pro-proliferative effect of AGK might be linked to activation of the AKT signaling pathway and subsequent inhibition of FOXO1 transcriptional activity, which would lead to altered expression of cell-cycle related genes, including the downregulation of CDK inhibitors, $p 21^{\text {Cip } 1} p 27^{\text {Kip1 }}$ and upregulation of cyclin D1. Our results have provided new insights into the role of AGK in the progression of human breast cancer, indicating that targeting AGK may offer a 


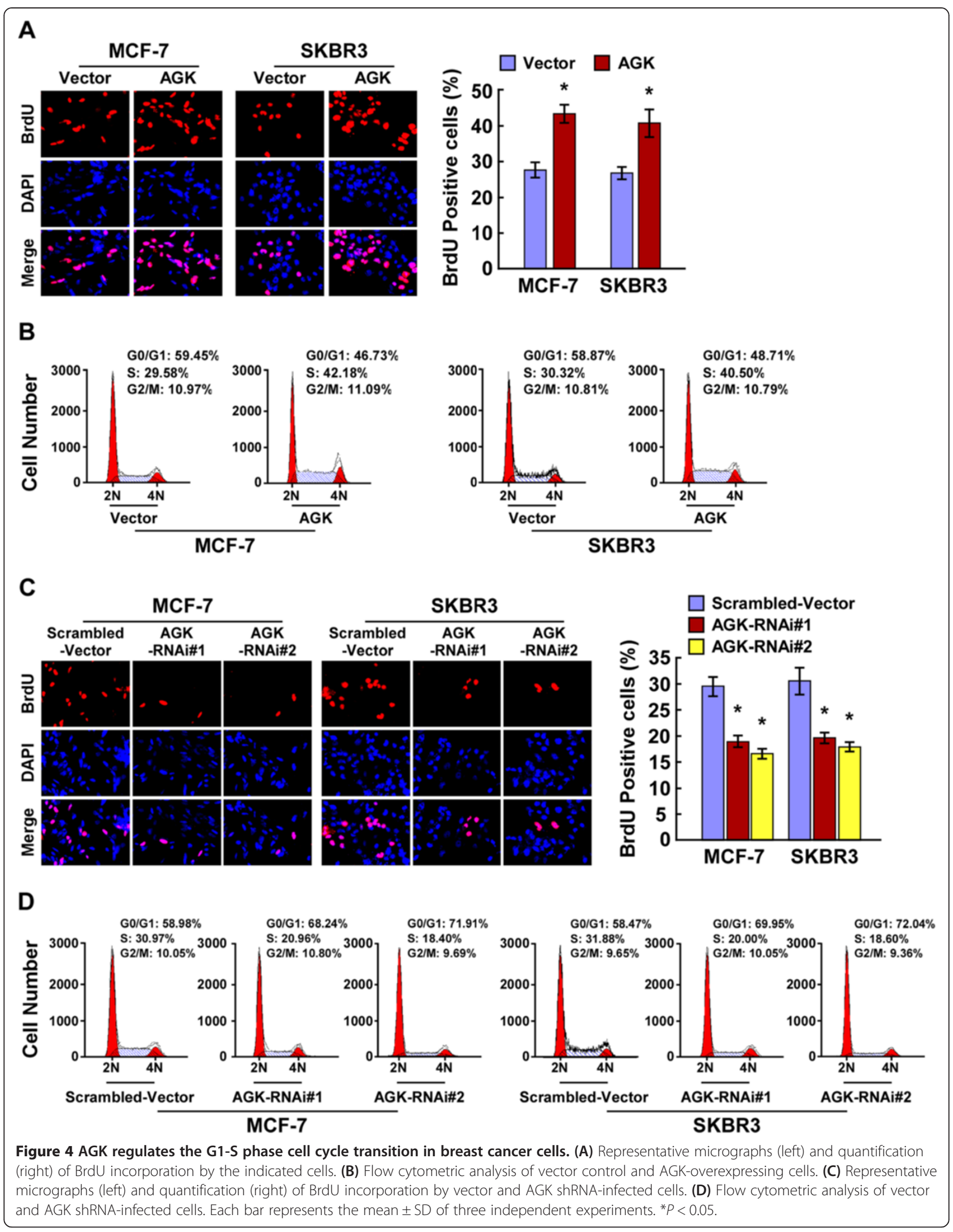




\section{A}

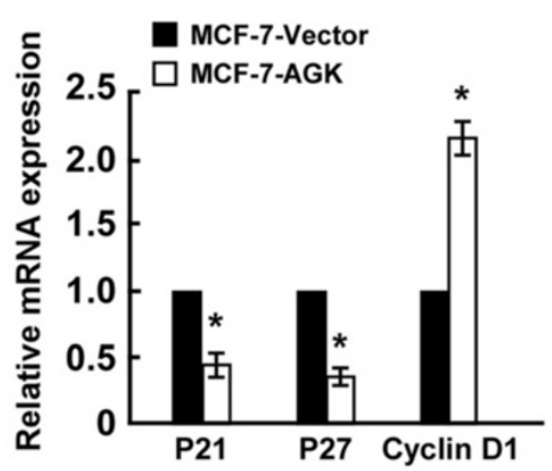

B

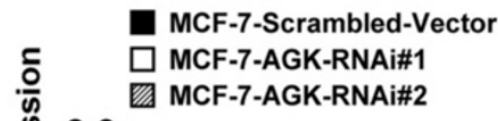

C

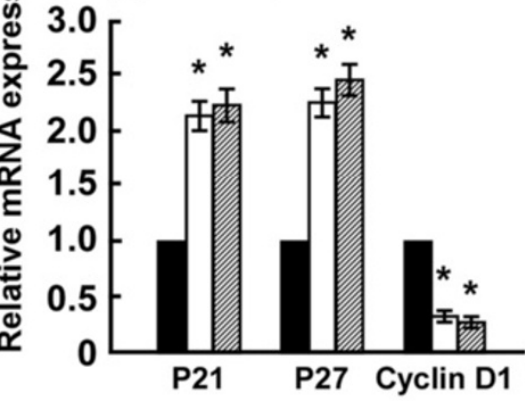

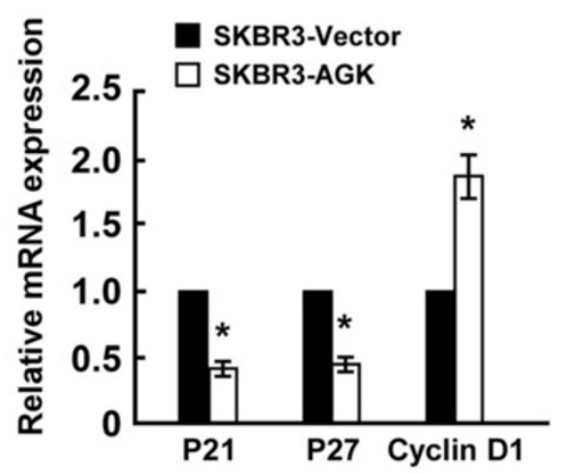

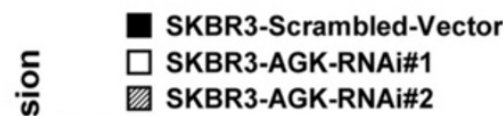

을 3.0
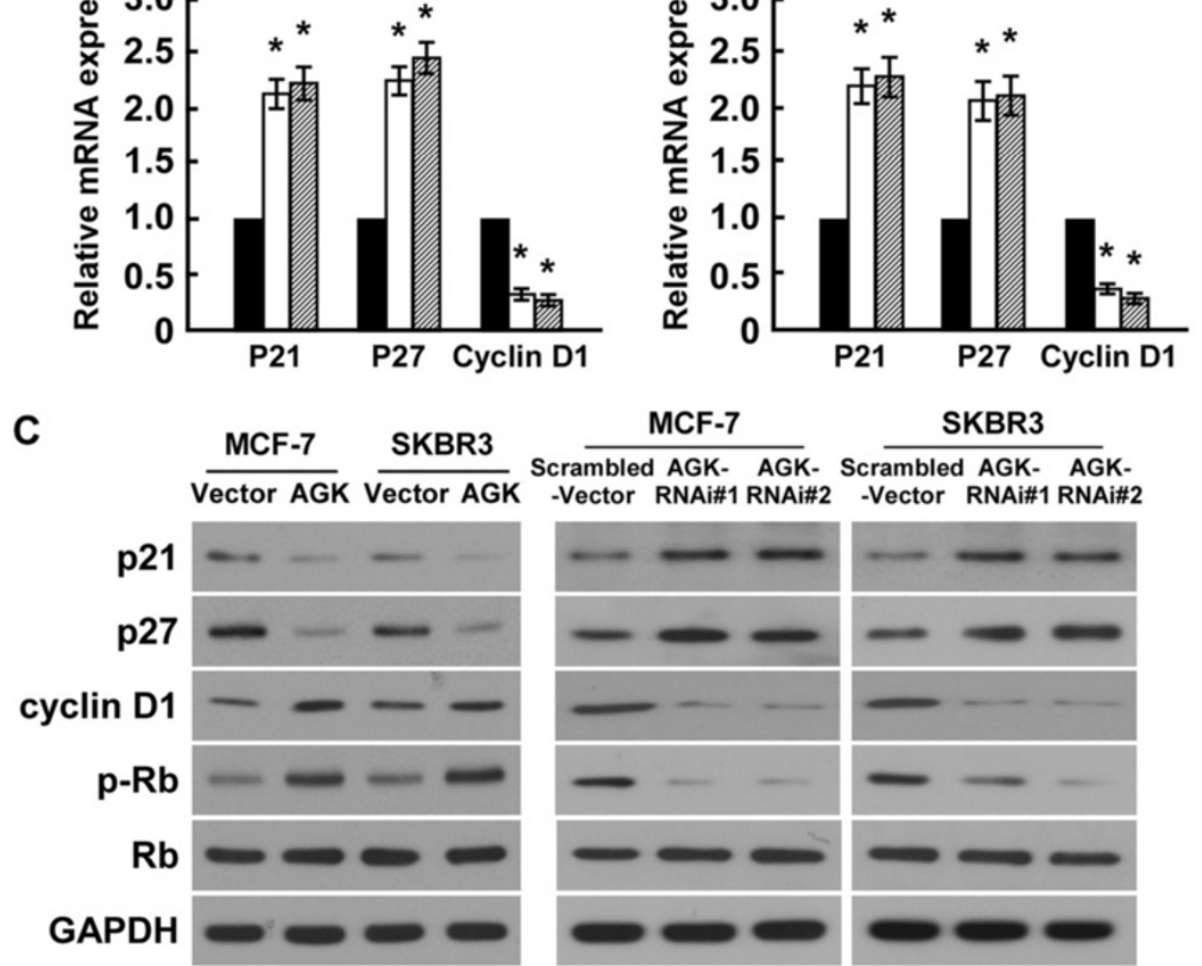

SKBR3

Scrambled AGK- AGK-

-Vector RNAi\#1 RNAi\#2

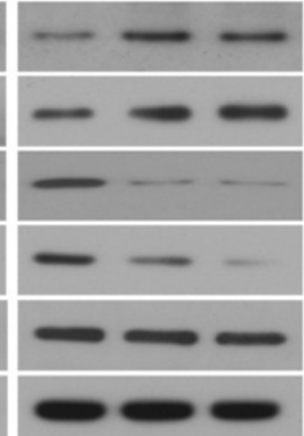

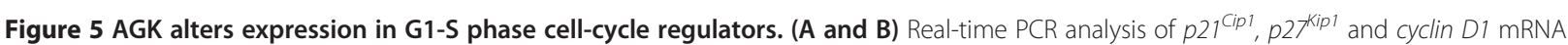
expression in AGK-infected cells (A) or AGK-shRNA infected cells (B). Gene expression levels were normalized to GAPDH. (C) Western blotting analysis of p21 ${ }^{\mathrm{Cip} 1}$, p27 ${ }^{\mathrm{Kip} 1}$, cyclin D1, p-Rb and total Rb protein expression in AGK-infected cells and AGK-shRNA infected cells; GAPDH was used as a loading control. Each bar represents the mean \pm SD of three independent experiments. ${ }^{*} P<0.05$.

novel therapeutic strategy in the treatment of patients with breast cancer.

Although AGK has been shown to be overexpressed in several types of cancer and has been associated with cancer progression and development [19,26,27], its clinical significance and biological role in human breast cancer remains unclear. In this study, we found that AGK expression was upregulated in a large cohort of human breast cancer tissues, and was significantly correlated with the clinicopathologic characteristics of breast cancer, including clinical stage and TNM classification. Furthermore, survival analyses showed that patients with higher levels of AGK expression had shorter overall survival time compared to those with lower AGK expression, suggesting that AGK may represent a novel predictor for prognosis and survival in breast cancer. Several reports have also provided evidence that AGK is involved in the survival and motility of malignant phenotypes of cancer cells and 


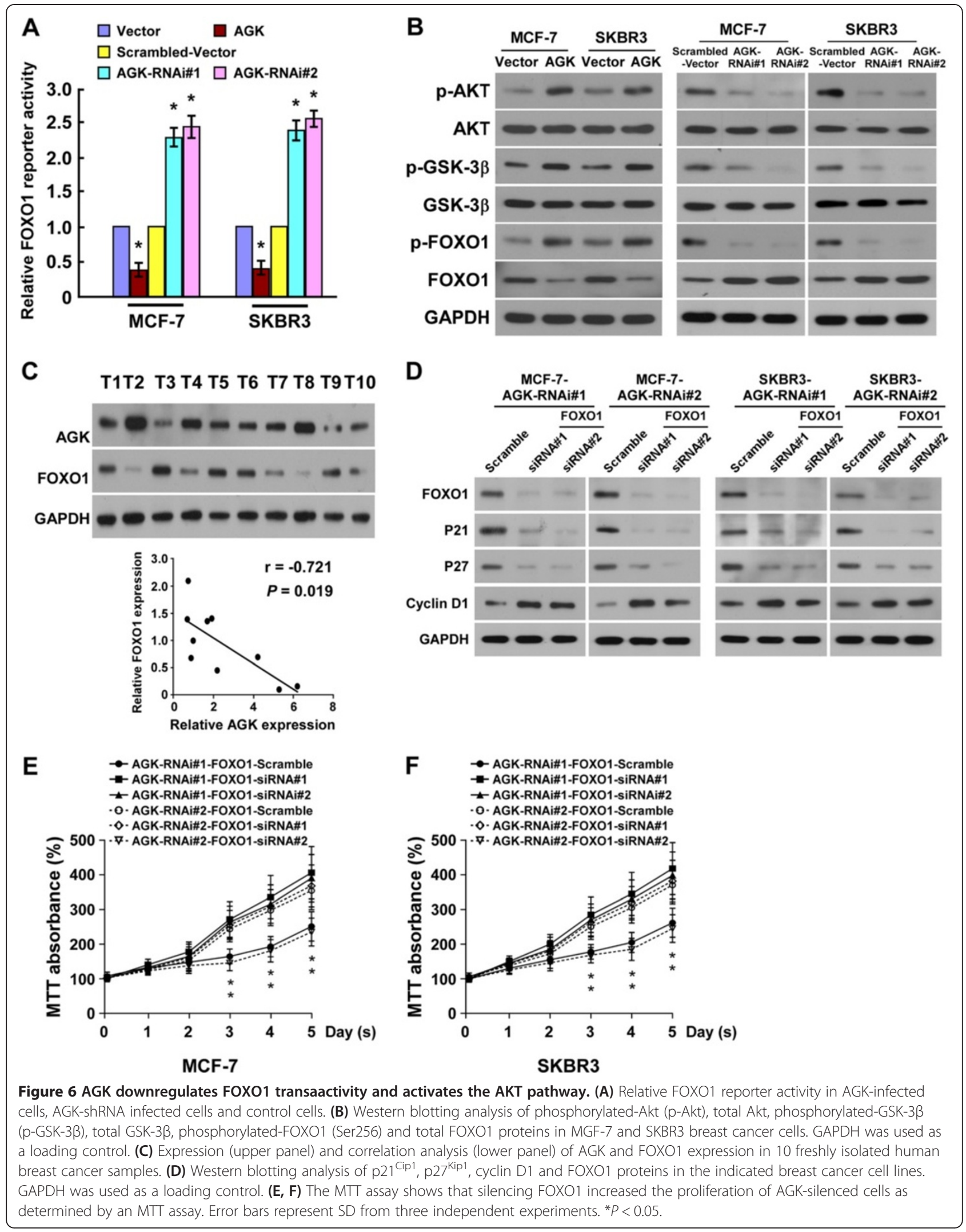


the development of cancer stem cells $[19,26]$. In agreement with these reports, we found that AGK was strongly expressed in highly proliferative lesions of human breast cancer, as indicated by a significant correlation between AGK and Ki-67 expression $(P<0.001)$. Combined with our observation that upregulation of AGK was implicated in the proliferation and tumorigenicity of breast cancer cells both in vivo and in vitro. Therefore, our results support the proposal that AGK is a proliferation-promoting and oncogenic protein in breast cancer cells.

It has been established that FOXO1 functions as a tumor suppressor. Consistently, FOXO1 expression is reported to be downregulated in multiple cancer types, such as breast cancer, prostate cancer, glioblastoma and endometrial carcinoma [15-18]. FOXO1 has been demonstrated to be involved in multiple biological processes through transcriptional regulation of their downstream efforts, such as CDK inhibitors $p 21^{\text {Cip } 1}, p 27^{\text {Kip } 1}$ and $p 57^{k i p 2}$, cell-cycle related genes cyclin $D 1 / D 2$, and proapoptotic proteins, such as Puma, Bim, TRAIL and FasL $[7-13,28,29]$. We showed that depletion of FOXO1 restored the growth rate of $A G K$-silenced breast cancer cells, indicating that FOXO1 plays a critical role in the proproliferative effect of AGK on breast cancer cells.

By showing that AGK overexpression decreased the transactivation of FOXO1 and its downstream targets, we hypothesized that FOXO1 was involved in AGKmediated proliferative mechanisms. It has been reported that phosphorylation of FOXO1 $\left(\mathrm{Ser}^{256}\right)$ by AKT results in downregulation of FOXO1 transactivity via ubiquitinproteasome-mediated degradation [30-32]. We observed similar effects, in that the levels of phospho-AKT and phospho-FOXO1 were increased in AGK-overexpressing cells and decreased in AGK-silenced cells. This suggested that the mechanism underlying AGK-mediated FOXO1 downregulation might be through activation of $\mathrm{AKT}$. AKT is a major downstream effector of epidermal growth factor receptor EGFR and the non-receptor tyrosine kinase JAK2 [33,34]. Interestingly, it has been reported that AGK overexpression promotes aggressiveness in prostate cancer cells through activation of EGFR, and that upregulation of AGK promotes the stem cell-like phenotype in ESCC by sustaining JAK2 activity [15,22]. Meanwhile, we also observed that the phosphorylation level of GSK-3 $\beta$, a downstream target protein of Akt, increased in the AGK-overexpressing cells and decreased in the AGK silenced cells. It has been reported that inactivation of GSK3 $\beta$ indicated by increased p-GSK3 $\beta$ was found in approximately half of the invasive mammary carcinomas, and significantly correlated with a worse clinical outcome [35]. Phosphorylation mediated suppression of GSK3 $\beta$ promotes breast tumor initiation and metastasis, and reduced phosphorylation of GSK3 $\beta$ efficiently inhibit cancer stem cell-like phenotypes in breast cancer [36,37].
Therefore, the role of AGK-modulation of GSK-3 $\beta$ activity in breast cancer cells is currently under investigation by our group.

\section{Conclusions}

In summary, our results have demonstrated that AGK plays an important role in human breast cancer progression and have provided insights into the underlying mechanisms. Establishing the precise role played by AGK in breast cancer progression will not only advance our understanding of the biology of breast cancer but may offer a mechanism for a novel therapeutic strategy via suppression of AGK expression in breast cancer cells. Furthermore, our results suggest a potential role for AGK as a clinical predictor of disease progression, prognosis and survival in patients with breast cancer. Evaluating the molecular diagnostic ability of AGK in breast cancer is merited.

\section{Methods \\ Cell lines}

Primary normal breast epithelial cells (NBECs) were established as previously described [38]. Breast cancer cell lines, including MCF-7, BT-549, ZR-75-1, SKBR3, MDA-MB-468, MDA-MB-435, Bcap37, MDA-MB-415, MDA-MB-361, T47D, MDA-MB-231 and ZR-75-30 were cultured in DMEM medium (Gibco, Grand Island, NY) supplemented with 10\% FBS (HyClone, Logan, UT).

\section{Patient information and tissue specimens}

This study was conducted on a total of 203 paraffinembedded, archived breast cancer samples, which had been histopathologically and clinically diagnosed at the Sun Yat-sen University Cancer Center from 1998 to 2006. Clinical and clinicopathological classification and stage were determined according to American Joint Committee on Cancer (AJCC) criteria [39] and summarized in Additional file 2: Table S1. Ethics approval and prior patient consent had been obtained from the Institutional Research Ethics Committee for the use of the clinical specimens for research purposes.

\section{Vectors and retroviral infection}

The human AGK gene was PCR-amplified from cDNA and cloned into the pSin-EF2 lentiviral vector, and shRNAs targeting AGK were cloned into the pSuperretro viral vector, as previously described [26]. Retroviral production and infection were performed as previously described [40]. Stable cell lines expressing AGK or AGK shRNAs were selected for 10 days with $0.5 \mu \mathrm{g} / \mathrm{ml}$ puromycin. The reporter plasmid for detecting the transcriptional activity of FOXO1 was generated as described previously [41]. 


\section{Immunohistochemistry (IHC)}

Immunohistochemistry (IHC) and quantification of AGK expression were performed by two independent pathologists, as previously described [26]. Both sets of results were combined to give a mean score for further comparative evaluations. Briefly, the IHC score, or staining index (SI), was determined by combining the score for the percentage of positively-stained tumor cells with the grade of the staining intensity. The percentages of positively-stained tumor cells were scored as follows: 0, no positive tumor cells; $1,<10 \%$; $2,10 \%-35 \%$; $3,35 \%-$ $75 \% ; 4,>75 \%$. The staining intensities were graded as follows: 1, no staining; 2, weak staining (light yellow); 3 , moderate staining (yellow-brown); 4, strong staining (brown). We used this method to evaluate AGK expression in benign breast epithelia and malignant lesions. The possible scores were $0,2,3,4,6,8,9,12$ and 16; SI $\geq 8$ was defined as high expression and SI $<8$ was defined as low expression.

\section{Western blotting}

Western blotting was carried out according to standard methods as described previously [38], by using anti-AGK antibody (Epitomics, Burlingame, CA), anti-p21 ${ }^{\mathrm{Cip} 1}$, antip27 ${ }^{\mathrm{Kip} 1}$, anti-cyclin $\mathrm{D} 1$, anti-Rb, anti-phosphorylated-Rb, anti-AKT, anti-phosphorylated-AKT, anti-FOXO1, antiphosphorylated-FOXO1 (Ser256) (Cell Signaling, Danvers, MA). The membranes were stripped and re-probed with an anti-GAPDH antibody (Sigma, Saint Louis, MI) as a loading control.

\section{MTT cell viability assay}

Cells were seeded in 96-well plates at a density of $2 \times 10^{3}$ cells/well. At each time point, cells were stained with $100 \mu \mathrm{l}$ sterile MTT dye $(0.5 \mathrm{mg} / \mathrm{ml}$, Sigma $)$ for 4 hours at $37^{\circ} \mathrm{C}$, followed by removal of the culture medium and addition of $100 \mu \mathrm{l}$ of dimethyl sulphoxide (Sigma). The absorbance was measured at $570 \mathrm{~nm}$, with $655 \mathrm{~nm}$ as the reference wavelength. The absorbance at day $1-5$ was normalized to the absorbance at day 0 used as control (100\%). Each experiment was performed in triplicates.

\section{Colony formation assay}

Cells were plated in 6-well plated $\left(5 \times 10^{2}\right.$ cells $)$ and cultured for 10 days. The colonies were stained with $1 \%$ crystal violet for 30 seconds after fixation with $4 \%$ formaldehyde for 5 minutes. Colonies were counted and the resultes were shown as the fold change compared to vector control cells.

\section{Anchorage-independent growth ability assay}

Five hundred cells were trypsinized and suspended in $2 \mathrm{ml}$ complete medium plus $0.3 \%$ agar (Sigma, Saint Louis, MI). The agar-cell mixture was plated on top of a bottom layer with $1 \%$ agar completed medium mixture. About 10 days, viable colonies that were larger than $0.1 \mathrm{~mm}$ were counted. The experiment was carried out for each cell line in triplicates.

\section{Bromodeoxyuridine (BrdU) labeling and immunofluorescence}

Cells $\left(5 \times 10^{4}\right)$ were plated on coverslips. After 24 hours, cells were incubated with BrdU for $1 \mathrm{~h}$ and stained with anti-BrdU antibody (Upstate, Billerica, MA) according to the manufacturer's instruction. After washing three times with PBS containing 1\% Triton X-100, the cells were treated with anti-mouse TRITC fluorescent conjugated secondary antibodies to visualize anti-BrdU labeled cells. BrdU positive cells were counted under a laser scanning microscope (Axioskop 2 plus; Carl Zeiss Co. Ltd.) in ten random chosen fields from three independent samples. Percentage of BrdU positive cells was then calculated, and the results are presented as the mean $\pm \mathrm{SD}$.

\section{Flow cytometry}

Cells were harvested, washed with cold PBS, and processed for cell cycle analysis by using flow cytometry. Briefly, the cells were fixed in $75 \%$ ethanol and stored at $-20^{\circ} \mathrm{C}$ overnight for later analysis. The fixed cells were centrifuged at 1,000 rpm for $5 \mathrm{~min}$ and washed with cold PBS twice. RNase A $(20 \mu \mathrm{g} / \mathrm{ml}$ final concentration) and propidium iodide staining solution $(50 \mu \mathrm{g} / \mathrm{ml}$ final concentration) were added to the cells and incubated for 30 minutes at $37^{\circ} \mathrm{C}$ in the dark. Twenty thousand cells were analyzed by using a CytomicsTM FC 500 instrument (Beckman Coulter, USA) equipped with CXP software. Modfit LT 3.1 trial cell cycle analysis software was used to determine the percentage of cells in the different phases of the cell cycle.

\section{Xenografted tumor model}

Female BALB/c nude mice ( $5 \sim 6$ weeks of age, $18 \sim 20 \mathrm{~g}$ ) were purchased from the Slac-Jingda Laboratory Animal (Hunan, China), and were housed in barrier facilities on a 12-hour light/dark cycle. All experimental procedures were approved by the Institutional Animal Care and Use Committee of Sun Yat-sen University. The BALB/c nude mice were randomly divided into 4 groups ( $n=5$ /group). A $0.72 \mathrm{mg}$ E2 60-day release pellet (Innovative Research of America) was implanted subcutaneously on the dorsal side of each mouse 1 day before tumor cell implantation to support the growth of the estrogen-dependent MCF-7 cell derived tumors. For tumor cell implantation, MCF-7AGK or MCF-7-AGK-RNAi or their respective control cells $\left(2 \times 10^{6}\right)$ in $200 \mu \mathrm{l}$ of the mixture were injected into the mammary fat pads of mice. Tumors were examined twice weekly; length, width, and thickness measurements were obtained with calipers and tumor volumes were 
calculated. On day 26, animals were euthanized, and tumors were excised and weighed.

\section{Statistical analysis}

Statistical analyses were performed using the SPSS version 13.0 statistical software package. Statistical tests for data analysis included log rank test, $\chi 2$ test, spearman-rank correlation test and Student's 2-tailed t test. Multivariate statistical analysis was performed using a Cox regression model. Data represent mean \pm SD. A $P$-value $<0.05$ was considered statistically significant.

\section{Additional files}

Additional file 1: Figure S1. AGK is upregulated in breast cancer. (A-B) Real-time PCR analysis of AGK mRNA expression in NBECs and twelve breast cancer cell lines (A), and in matched primary breast cancer tissues (T) and the adjacent noncancerous tissues (N) (B). Expression levels were normalized to GAPDH. Error bars represent SD from three independent experiments. ${ }^{*} P<0.05$.

Additional file 2: Table S1. Clinicopathological characteristics of patient samples and expression of AGK in Breast Cancer. Table S2. Correlation between AGK expression and clinicopathologic characteristics of Breast Cancer. Table S3. Univariate and multivariate analyses of various prognostic parameters in patients with Breast cancer Cox-regression analysis.

Additional file 3: Figure S2. (A-B) Kaplan-Meier overall survival curves and log-rank test in breast cancer patients stratified by clinical stage I-II $(n=131)(\mathbf{A})$ and clinical stage III-IV $(n=72)$ (B). The curves compare patients with high and low expression levels of AGK.

Additional file 4: Figure S3. FOXO1 plays an important role in AGK-mediated proliferation. (A) Relative FOXO1 reporter activity in AGK-silenced MCF-7 and MDA-MB-231 cells after transfection with FOXO1 siRNA(s). (B) Real-time PCR analysis of p21 ${ }^{\mathrm{Cip} 1}, \mathrm{p} 27^{\mathrm{Kip} 1}$ and cyclin D1 mRNA expression in AGK-silenced MCF-7 and MDA-MB-231 cells after transfection with FOXO1 siRNA(s). Expression levels were normalized to GAPDH. (C) Quantification of colony formation assays in indicated cells. Error bars represent SD from three independent experiments. ${ }^{*} P<0.05$.

\section{Abbreviations}

AGK: Acylglycerol kinase; IHC: Immunochemistry; TNM: Tumor-nodulemetastasis; FOXO: Forkhead box-containing O subfamily; FASL: Fas ligand; LPA: Lysophosphatidic acid; OS: Overall survival; CDK: Cyclin dependent kinases; NBECs: Normal breast epithelial cells; BrdU: Bromodeoxyuridine.

\section{Competing interests}

The authors declare that they have no competing interests.

\section{Authors' contributions}

$X W, C L$ and $X Z$ were responsible for most experiments, data collection and analysis. AL and JZ were responsible for conducting the data analysis and Luciferase assay. XL was responsible for Real-time PCR assay. LS was responsible for experimental design, supervised the project and wrote the manuscript. All authors have read and approved the final manuscript.

\section{Acknowledgements}

Supported by the Ministry of Science and Technology of China grant (No. 973-2014CB910604); The Natural Science Foundation of China (81272198, U1201121, 81171892); The Science and Technology Department of Guangdong Province, China (S2011020002757); Guangdong Provincial Department of Science and Technology (2010B031600219); Ministry of Education of China (20100171110080).

\section{Author details}

${ }^{1}$ State Key Laboratory of Oncology in Southern China, Department of Experimental Research, Sun Yat-sen University Cancer Center, Guangzhou, Guangdong 510060, China. ²Department of Breast Surgery, Cancer Center, Sun Yat-sen University, Guangzhou, Guangdong 510060, China. ${ }^{3}$ Department of Biochemistry, Zhongshan School of Medicine, Sun Yat-sen University, Guangzhou, Guangdong 510080, China.

Received: 11 December 2013 Accepted: 22 April 2014

Published: 8 May 2014

\section{References}

1. Jemal A, Bray F, Center MM, Ferlay J, Ward E, Forman D: Global cancer statistics. CA Cancer J Clin 2011, 61:69-90.

2. Guo L, Cui ZM, Zhang J, Huang Y: Chemokine axes CXCL12/CXCR4 and CXCL16/CXCR6 correlate with lymph node metastasis in epithelial ovarian carcinoma. Chin J Cancer 2011, 30:336-343.

3. Zeng Z, Lin H, Zhao X, Liu G, Wang X, Xu R, Chen K, Li J, Song L: Overexpression of GOLPH3 promotes proliferation and tumorigenicity in breast cancer via suppression of the FOXO1 transcription factor. Clin Cancer Res 2012, 18:4059-4069.

4. Lu Z: Nonmetabolic functions of pyruvate kinase isoform M2 in controlling cell cycle progression and tumorigenesis. Chin J Cancer 2012 31:5-7.

5. Arden KC: FoxOs in tumor suppression and stem cell maintenance. Cell 2007, 128:235-237.

6. Paik JH, Kollipara R, Chu G, Ji H, Xiao Y, Ding Z, Miao L, Tothova Z, Horner JW, Carrasco DR, Jiang S, Gilliland DG, Chin L, Wong WH, Castrillon DH, DePinho RA: FoxOs are lineage-restricted redundant tumor suppressors and regulate endothelial cell homeostasis. Cell 2007, 128:309-323.

7. Medema RH, Kops GJ, Bos JL, Burgering BM: AFX-like Forkhead transcription factors mediate cell-cycle regulation by Ras and PKB through p27kip1. Nature 2000, 404:782-787

8. Kops GJ, Medema RH, Glassford J, Essers MA, Dijkers PF, Coffer PJ, Lam EW, Burgering BM: Control of cell cycle exit and entry by protein kinase B-regulated forkhead transcription factors. Mol Cell Biol 2002, 22:2025-2036.

9. Schmidt M, Fernandez de Mattos S, van der Horst A, Klompmaker R, Kops GJ, Lam EW, Burgering BM, Medema RH: Cell cycle inhibition by FoxO forkhead transcription factors involves downregulation of cyclin D. Mol Cell Biol 2002, 22:7842-7852.

10. You H, Pellegrini M, Tsuchihara K, Yamamoto K, Hacker G, Erlacher M, Villunger A, Mak TW: FOXO3a-dependent regulation of Puma in response to cytokine/growth factor withdrawal. J Exp Med 2006, 203:1657-1663.

11. Dijkers PF, Medema RH, Lammers JW, Koenderman L, Coffer PJ: Expression of the pro-apoptotic Bcl-2 family member Bim is regulated by the forkhead transcription factor FKHR-L1. Curr Biol 2000, 10:1201-1204.

12. Yang JY, Xia W, Hu MC: lonizing radiation activates expression of FOXO3a, Fas ligand, and Bim, and induces cell apoptosis. Int J Oncol 2006, 29:643-648.

13. Modur V, Nagarajan R, Evers BM, Milbrandt J: FOXO proteins regulate tumor necrosis factor-related apoptosis inducing ligand expression. Implications for PTEN mutation in prostate cancer. J Biol Chem 2002, 277:47928-47937.

14. Furukawa-Hibi Y, Yoshida-Araki K, Ohta T, Ikeda K, Motoyama N: FOXO forkhead transcription factors induce $\mathrm{G}$ (2)-M checkpoint in response to oxidative stress. J Biol Chem 2002, 277:26729-26732.

15. Dong XY, Chen C, Sun X, Guo P, Vessella RL, Wang RX, Chung LW, Zhou W, Dong JT: FOXO1A is a candidate for the $13 q 14$ tumor suppressor gene inhibiting androgen receptor signaling in prostate cancer. Cancer Res 2006, 66:6998-7006

16. Ward EC, Hoekstra AV, Blok LJ, Hanifi-Moghaddam P, Lurain JR, Singh DK, Buttin BM, Schink JC, Kim JJ: The regulation and function of the forkhead transcription factor, Forkhead box 01 , is dependent on the progesterone receptor in endometrial carcinoma. Endocrinology 2008, 149:1942-1950.

17. Seoane J, Le HV, Shen L, Anderson SA, Massagué J: Integration of Smad and forkhead pathways in the control of neuroepithelial and glioblastoma cell proliferation. Cell 2004, 117:211-223.

18. Guttilla IK, White BA: Coordinate regulation of FOXO1 by miR-27a, miR-96, and miR-182 in breast cancer cells. J Biol Chem 2009, 284:23204-23216. 
19. Bektas M, Payne SG, Liu H, Goparaju S, Milstien S, Spiegel S: A novel acylglycerol kinase that produces lysophosphatidic acid modulates cross talk with EGFR in prostate cancer cells. J Cell Biol 2005, 169:801-811.

20. Epand RM, Shulga YV, Timmons HC, Perri AL, Belani JD, Perinpanathan K, Johnson-McIntire LB, Bajjalieh S, Dicu AO, Elias C, Rychnovsky SD, Topham MK: Substrate chirality and specificity of diacylglycerol kinases and the multisubstrate lipid kinase. Biochemistry 2007, 46:14225-14231.

21. Zhao Y, Natarajan V: Lysophosphatidic acid signaling in airway epithelium: role in airway inflammation and remodeling. Cell Signal 2009, 21:367-377.

22. Kalari S, Zhao Y, Spannhake EW, Berdyshev EV, Natarajan V: Role of acylglycerol kinase in LPA-induced IL-8 secretion and transactivation of epidermal growth factor-receptor in human bronchial epithelial cells. Am J Physiol Lung Cell Mol Physiol 2009, 296:328-336.

23. Kim JH, Adelstein RS: LPA (1)-induced migration requires nonmuscle myosin II light chain phosphorylation in breast cancer cells. J Cell Physiol 2011, 226:2881-2893.

24. Swamydas M, Nguyen D, Allen LD, Eddy J, Dréau D: Progranulin stimulated by LPA promotes the migration of aggressive breast cancer cells. Cell Commun Adhes 2011, 18:119-130

25. Du J, Sun C, Hu Z, Yang Y, Zhu Y, Zheng D, Gu L, Lu X: Lysophosphatidic acid induces SKBR3 breast cancer cells migration through activation of PI3K/PAK1/ERK signaling. PLOS One 2010, 5:e15940.

26. Chen $X$, Ying $Z$, Lin $X$, Lin H, Wu J, Li M, Song L: Acylglycerol kinase augments JAK2/STAT3 signaling in esophageal squamous cells. J Clin Invest 2013, 123:2576-2589.

27. Nouh MA, Wu XX, Okazoe H, Tsunemori H, Haba R, Abou-Zeid AM, Saleem MD, Inui M, Sugimoto M, Aoki J, Kakehi Y: Expression of autotaxin and acylglycerol kinase in prostate cancer: association with cancer development and progression. Cancer Sci 2009, 100:1631-1638.

28. Nakamura N, Ramaswamy S, Vazquez F, Signoretti S, Loda M, Sellers WR: Forkhead transcription factors are critical effectors of cell death and cell cycle arrest downstream of PTEN. Mol Cell Biol 2000, 20:8969-8982.

29. Roeb W, Boyer A, Cavenee WK, Arden KC: PAX3-FOXO1 controls expression of the $\mathrm{p} 57^{\mathrm{Kip} 2}$ cell-cycle regulator through degradation of EGR1. Proc Natl Acad Sci U S A 2007, 104:18085-18090.

30. Cardozo T, Pagano M: The SCF ubiquitin ligase: insights into a molecular mechanism. Nat Rev Mol Cell Biol 2004, 5:739-753.

31. Masahiro A, Hao J, Peter KV: Proteasomal degradation of the FOXO1 transcriptional regulator in cells transformed by the PI3K and AKT oncoproteins. Proc Natl Acad Sci U S A 2004, 101:13613-13617.

32. Huang $H$, Regan KM, Wang F, Wang D, Smith DI, van Deursen JM, Tindall DJ: Skp2 inhibits FOXO1 in tumor suppression through ubiquitin-mediated degradation. Proc Natl Acad Sci U S A 2005 102:1649-1654.

33. Vivanco I, Sawyers CL: The phosphatidylinositol 3-Kinase AKT pathway in human cancer. Nat Rev Cancer 2002, 2:489-501.

34. Tokunaga E, Oki E, Egashira A, Sadanaga N, Morita M, Kakeji Y, Maehara Y: Deregulation of the AKT pathway in human cancer. Curr Cancer Drug Targets 2008, 8:27-36.

35. Armanious H, Deschenes J, Gelebart P, Ghosh S, Mackey J, Lai R: Clinical and biological significance of GSK-3 $\beta$ inactivation in breast cancer-an immunohistochemical study. Hum Pathol 2010, 41:1657-1663.

36. Takahashi RU, Takeshita F, Honma K, Ono M, Kato K, Ochiya T: Ribophorin II regulates breast tumor initiation and metastasis through the functional suppression of GSK3 $\beta$. Sci Rep 2013, 3:2474

37. Li Y, Zhang T, Korkaya H, Liu S, Lee HF, Newman B, Yu Y, Clouthier SG, Schwartz SJ, Wicha MS, Sun D: Sulforaphane, a dietary component of broccoli/broccoli sprouts, inhibits breast cancer stem cells. Clin Cancer Res 2010, 16:2580-2590.

38. Li J, Zhang N, Song LB, Liao WT, Jiang LL, Gong LY, Wu J, Yuan J, Zhang HZ, Zeng MS, Li M: Astrocyte elevated gene- 1 is a novel prognostic marker for breast cancer progression and overall patient survival. Clin Cancer Res 2008, 14:3319-3326.

39. Edge S, Byrd DR, Compton CC, Fritz AG, Greene FL, Trotti A: Breast Cancer in AJCC Cancer Staging Handbook. TNM Classification of Malignant Tumors. 7th edition. New York: Springer Verlag; 2010:255-281.
40. Hahn WC, Dessain SK, Brooks MW, King JE, Elenbaas B, Sabatini DM, DeCaprio JA, Weinberg RA: Enumeration of the simian virus 40 early region elements necessary for human cell transformation. Mol Cell Biol 2002, 22:2111-2123.

41. Tang ED, Nuñez G, Barr FG, Guan KL: Negative regulation of the forkhead transcription factor FKHR by AKT. J Biol Chem 1999, 274:16741-16746

doi:10.1186/1476-4598-13-106

Cite this article as: Wang et al:: Acylglycerol kinase promotes cell proliferation and tumorigenicity in breast cancer via suppression of the FOXO1 transcription factor. Molecular Cancer 2014 13:106.

\section{Submit your next manuscript to BioMed Central and take full advantage of:}

- Convenient online submission

- Thorough peer review

- No space constraints or color figure charges

- Immediate publication on acceptance

- Inclusion in PubMed, CAS, Scopus and Google Scholar

- Research which is freely available for redistribution

Submit your manuscript at www.biomedcentral.com/submit
C Biomed Central 